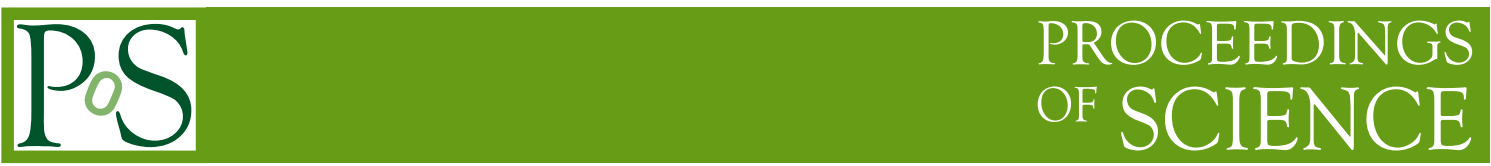

\title{
WIMP neutrinos from the Sun and the Earth $\left(15^{\prime}+5^{\prime}\right)$
}

\section{MATTIAS BLENNOW*}

Max-Planck-Institut für Physik

E-mail: blennow@mppmu.mpg.de

\section{JOAKIM EDSJÖ}

Department of Physics, Stockholm University

E-mail: eds jo@physto.se

\section{TOMMY OHLSSON}

Department of Theoretical Physics, Royal Institute of Technology

E-mail: tommyatheophys.kth.se

We discuss the propagation treatment of the indirect dark matter detection using WIMP annihilations in the Sun and the Earth. In particular, we focus on treating neutrino interactions and oscillations in a consistent framework, including tau neutrino regeneration and a full three-flavor neutrino oscillation framework. We also discuss the equivalence of using a Monte Carlo approach - suited for inclusion in neutrino telescope Monte Carlos - and the density matrix formalism.

Identification of dark matter 2008

August 18-22, 2008

Stockholm, Sweden

\footnotetext{
* Speaker.
} 


\section{Introduction}

When weakly massive interactive particles (WIMPs) from the galactic halo passes through the Sun, there is a possibility that they scatter and lose enough energy to be gravitationally trapped. Following subsequent scatterings in the Sun, the WIMPs sink to the solar core and thermalize. The accreted WIMPs can then undergo co-annihilation into different channels depending on the particular WIMP model. Regardless of the model, the only particles which interact weakly enough to escape from the Sun are neutrinos, which can be produced either directly or through the subsequent decays of the co-annihilation products. Here, we will present an event based Monte Carlo for computing the neutrino flux at Earth given the co-annihilation rate and branching fractions into the different channels. We will also comment on the equivalence between this Monte Carlo and the density matrix formalism usually used to treat this phenomenon analytically.

\section{Density matrix formalism}

When treating the neutrino propagation analytically, it is customary to use the density matrix formalism in which the neutrino evolution is given by

$$
\dot{\rho}(r, E)=-\mathrm{i}[H, \rho]-\frac{1}{2}\{\Gamma, \rho\}+\dot{\rho}_{\mathrm{reg}},
$$

where $\rho(r, E)$ is describing the flavor and energy distribution of neutrinos, the first term on the right-hand side describes neutrino oscillations, the second describes the degrading of the neutrino flux due to interactions, and the last term is a regeneration term describing the gain in neutrinos from the same interactions (a neutral-current interaction simply degrades the neutrino energy, while a charged-current interaction may produce new neutrinos if the neutrino interacts as a $v_{\tau}$ ). This approach has been used in a number of papers in order to study the impact of neutrino oscillations and interactions in a consistent framework [2, 3, 4].

\section{Monte Carlo treatment}

In our Monte Carlo, we instead use an event based approach, where every neutrino is described by a vector in flavor space as well as an energy. We then compute if and when the neutrino will interact. If the neutrino does not interact, then we simply evolve its flavor state any desired point. However, if it does interact, it is treated differently depending on the type of interaction. For neutral current interactions, we simply remove the neutrino from our simulation and create a new neutrino with the same flavor vector but degraded energy due to the energy loss in the interaction. If the interaction is due to a charged-current, then the neutrino is completely lost unless it interacts as a $v_{\tau}$. In this latter case, the resulting $\tau$ will decay before it loses energy due to interactions, and thus, produce new neutrinos. We then simulate this interaction and subsequent decay and add the resulting neutrinos to our computation.

It is fairly straightforward to show that this Monte Carlo treatment is equivalent to the usual density matrix formalism on a statistical level. The simplest way of doing this is to start from the fact that the evolution of the density matrix is linear (this is no longer true if one considers the evolution where neutrino-neutrino interactions become important, such as above the neurino sphere 


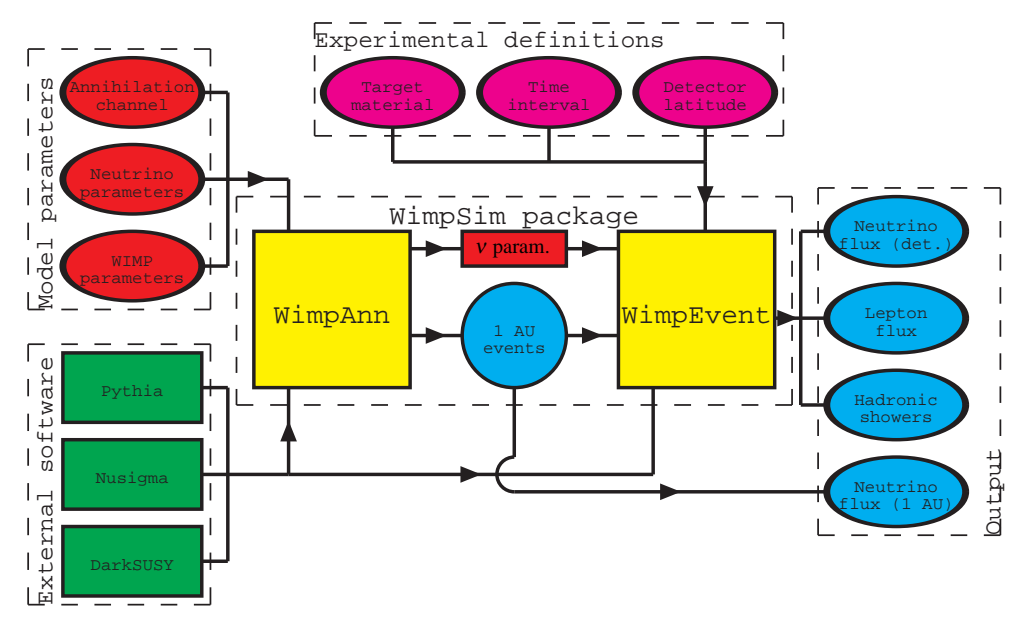

Figure 1: A flowchart of how the WimpSim package operates, including input, output and dependencies on external programs.

in a supernova). Then one can construct the density matrix corresponding to a single neutrino in the Monte Carlo and deduce how it is propagated. This readily gives back the density matrix evolution when one considers the statistical average. For details, see the Appendix of Ref. [1].

Our Monte Carlo has been written in a very general format and is easy to implement together with DarkSUSY or into neutrino telescope Monte Carlos. The code, as well as the results of our simulations are publically available [5]. Essentially, the code is built up of two main programs, WimpAnn and WimpEvent, which together form the program package WimpSim. WimpAnn is responsible for the treatment from the WIMP annihilations up to the propagation of the resulting neutrinos to a distance of $1 \mathrm{AU}$. Essentially, this is the part which needs no input on the experimental setup. From 1 AU, WimpEvent takes over and propagates the neutrinos to the detector and computes the final neutrino fluxes and other data that could be of interest. For a schematic view on how the WimpSim package operates, see Fig. 1. Further information can be found at Ref. [5].

\section{Results}

As our main example in Ref. [1], we present the results for annihilations of $m_{\chi}=250 \mathrm{GeV}$ WIMPs into $\tau^{+} \tau^{-}$pairs, simply because this channel will give a relatively large difference in the initial fluxes of different neutrino flavors. The difference between the simulations with and without neutrino oscillations included are shown in Fig. 2. Essentially, the results arise in the following manner: On the way out of the Sun, $v_{\mu} \leftrightarrow v_{\tau}$ oscillations due to the mass squared splitting $\Delta m_{31}^{2}$ more or less equilibrates the $v_{\mu}$ and $v_{\tau}$ fluxes, although the oscillation pattern can still be seen in the energy spectrum. When propagated to a distance of $1 \mathrm{AU}$, also the mass squared splitting $\Delta m_{21}^{2}$ has started to affect the oscillations and also produced an increased flux of $v_{e}$ at the expense of the other neutrino flavors. The $v_{\mu} \leftrightarrow v_{\tau}$ oscillations are now so fast that they are no longer seen in the energy spectrum, while there remains an oscillation pattern for the oscillations into $v_{e}$ at higher energies. Finally, the propagation to a detector (in this case a detector located at the South 


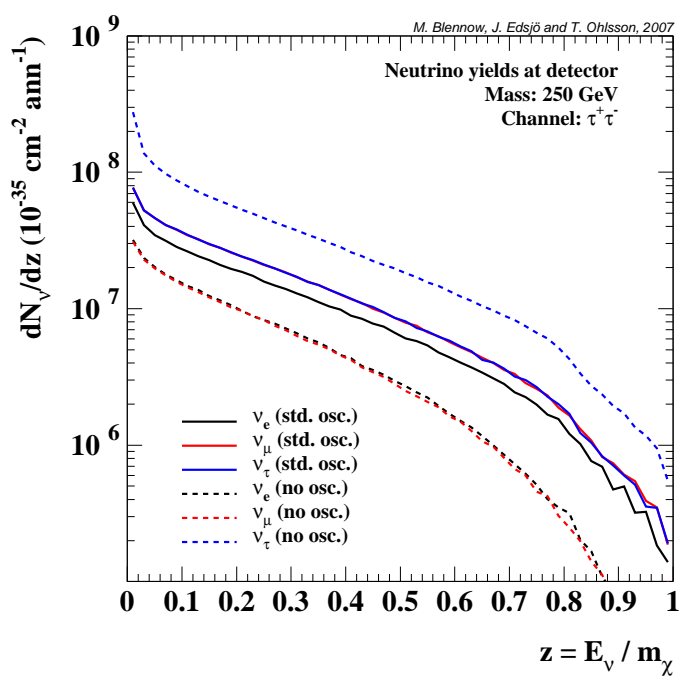

Figure 2: The result for annihilations of a $m_{\chi}=250 \mathrm{GeV}$ WIMP into $\tau^{+} \tau^{-}$, both in the case when neutrino oscillations are included (std osc.) and for the case when they are not (no osc.). Figure from Ref. [1].

Pole) and making a time-average over half a year essentially erases all of the remaining oscillation features (see Ref. [1] for details).

\section{Acknowledgments}

This work was supported by the Royal Swedish Academy of Sciences (KVA) [TO] and the Swedish Research Council (Vetenskapsrådet) through Contract Nos. 623-2007-8066 [MB], 6222003-6025 [JE] and 621-2005-3588 [TO].

\section{References}

[1] M. Blennow, J. Edsjö, and T. Ohlsson, Neutrinos from WIMP Annihilations Using a Full Three-Flavor Monte Carlo. JCAP 01 (2008) 021 [0709 . 3898].

[2] M. Cirelli et al., Spectra of neutrinos from dark matter annihilations, Nucl. Phys. 3727 (2005) 99 [hep-ph/0506298].

[3] R. Lehnert and T. J. Weiler, Neutrino flavor ratios as diagnostic of solar WIMP annihilation, Phys. Rev. D77 (2008) 125004 [0708.1035].

[4] V. Barger, W.-Y. Keung, G. Shaughnessy, and A. Tregre, High energy neutrinos from neutralino annihilations in the Sun, Phys. Rev. D76 (2007) 095008 [0 708 . 1325].

[5] http://www.physto.se/〜edsjo/wimpsim, WimpSim homepage. 